\title{
Influence of Exposure Energy Control for High Voltage Pulsed Xenon Lamp on the Lithography Accuracy of Linear Grating
}

\author{
Dongxu Ren ${ }^{1 *}$, Zexiang Zhao ${ }^{1}$, Bin $\mathrm{Li}^{1}$, Wenhan Zeng ${ }^{1}$ \\ 1. School of Mechatronics Engineering, \\ Zhongyuan University of Technology, \\ Zhengzhou, China \\ * rendongxu313@zut.edu.cn
}

\begin{abstract}
In this paper, an exposure energy analysis method which affects the precision of incremental grating lithography is proposed. Based on the analysis of the charge and discharge circuit of high voltage pulsed xenon lamp power supply, the change the characteristics of charge and discharge voltage and current circuits is calculated and analyzed by using the Thevenin's basic theorem. The complete charge and discharge voltage, circuit and exposure energy of the pulsed xenon discharge circuit were quantitatively analyzed. The grating energy model and grating fringes were established by calculating the lithography threshold model. Through the example analysis, in order to reduce the influence on the lithography energy, the resistance value of the pulsed xenon lamp should be controlled within $1 \Omega$, and the error of the exposure time delay is controlled within $2 \mathrm{~ms}$, which provides a theoretical basis for the lithography and control method of highprecision grating scale calculation.
\end{abstract}

Keywords-incremental linear grating; computational lithography; pulsed xenon lamp; linear scale accuracy; lithography energy model

\section{INTRODUCTION}

The manufacturing precision of the incremental grating has been widely concerned in the field of precision ultraprecision machining and measurement. Due to the different manufacturing principles, the influence parameters are also different. In the projection grating lithography process, the charge and discharge control system of high-voltage pulsed xenon lamp is one of the factors affecting the accuracy of the incremental grating ruler. It can emit a large amount of energy during the discharge process, the discharge process is short, the xenon lamp has a short flashing time, and has a high repeating switch. The discharge frequency can meet the requirements of dynamic lithography for illumination. The pulsed xenon lamp power control system is the main factor affecting the energy release time [1-3]. In raster computing lithography, the static exposure of the lithographic threshold model is used to calculate the accuracy of the exposure energy on the grating fringe [4-5], such as the dynamic exposure energy lithography of the diffraction grating [6]. In this paper, the volt-ampere characteristics of pulsed xenon lamp are studied for the pulsed xenon lamp power supply circuit. Based on the threshold model of static calculation lithography, the influence of pulse xenon lamp resistance and time delay error on the energy of the grating is studied.

\section{BASIC PRINCIPLE OF HIGH VOLTAGE PULSE CONTROL}

\section{A. The basic principle of pulsed xenon lamp}

A pulsed xenon lamp is a xenon lamp that uses stored electrical energy or chemical energy to generate a high intensity flash in a very short time. The luminescence characteristics of the pulsed xenon lamp, that is, the spectrum is determined by the ultraviolet xenon lamp driving power source.

As shown in the working principle of the pulsed xenon lamp in Fig. 1, the inside of the xenon lamp is full of helium gas, and the resistance at both ends of the xenon lamp in the free state is very high, which is quite open, and the capacitor $\mathrm{S}$ cannot be discharged when the switch $\mathrm{S}$ is closed. In order to enable the circuit to work normally, it is necessary to maintain the state of pre-ionization of the gas inside the xenon lamp, the resistance at both ends becomes small, and the closed capacitance of the switch S satisfies the high-voltage discharge condition.

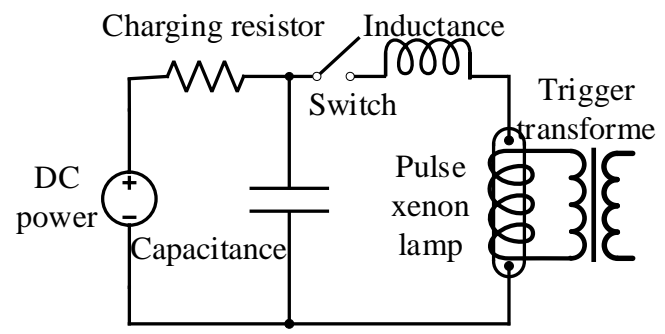

Figure 1.

The trigger transformer generates a high-voltage pulse to break down the helium gas in the xenon lamp, generally reaching a voltage of $10 \mathrm{kV}$ or higher, so that the gas inside the xenon lamp is in a pre-ionization state, which is called a start-up circuit, and the xenon lamp enters a pre-ionization state to maintain pre-ionization. The voltage required for the state is not high and does not require a large current. After the discharge of the capacitor is completed, the xenon lamp is powered by the circuit that maintains the preionization state of the xenon lamp. The capacitor does not need to be re-ignited for the next discharge; the xenon lamp can be repeatedly subjected to high voltage discharge, and each time the capacitor can pass The xenon lamp is fully

Supported by National Natural Science Foundation of China (Grant No.51475485), Open Laboratory of Key Disciplines of Modern Manufacturing Equipment and Instruments of Henan Higher Education Institutions (2016MEI005) and Young Talents Innovation Ability Fund Project of Zhongyuan University of Technology (K2018QN002). 
discharged, and there is no possibility that the capacitor cannot be discharged.

\section{B. Discharge control circuit}

In this paper, the circuit schematic diagram is shown in Figure 2. $U_{2}$ is the ignition voltage, $U_{3}$ is the voltage to maintain the pre-ionization state of the xenon lamp, and $U_{1}$ is the discharge voltage of the capacitor. The working process of the ultraviolet xenon lamp driving power supply is that first, the ignition voltage $\mathrm{U}_{2}$ charges the capacitor $\mathrm{C}_{2}$ through the resistor $\mathrm{R}_{4}$. When the thyristor $\mathrm{VT}_{2}$ is turned on, the capacitor $\mathrm{C}_{2}$ is discharged through the transformer, generating a sufficiently high voltage in the secondary of the transformer, and the xenon lamp The gas inside is ionized. Once the gas in the xenon lamp is ionized, its resistance becomes very small, so that voltage $U_{3}$ maintains the pre-ionization state of the xenon lamp through diode $\mathrm{D}$ and resistor $R_{3}$. Since the xenon lamp is already in an ionized state at this time, as long as the thyristor $\mathrm{VT}_{1}$ is turned on, the voltage on the capacitor $\mathrm{C}_{1}$ can be discharged by the xenon lamp. After the voltage on capacitor $C_{1}$ is discharged, voltage $\mathrm{U}_{2}$ then maintains the pre-ionization state of the xenon lamp until the next discharge.

The operation of the pulsed xenon lamp is divided into three stages: ignition, pre-combustion and high-voltage discharge. In the start-up phase, the discharge first generates an ionization channel at the inner wall of the quartz tube near the trigger wire. The gas is heated due to collision with the electron, and the helium gas in the lamp is rapidly ionized. A glow discharge occurs. In the precombustion phase, when the input energy is sufficiently large, the electrode is heated to have a certain thermal emission capability, and the gas in the lamp tube is converted from a glow discharge to an arc discharge.

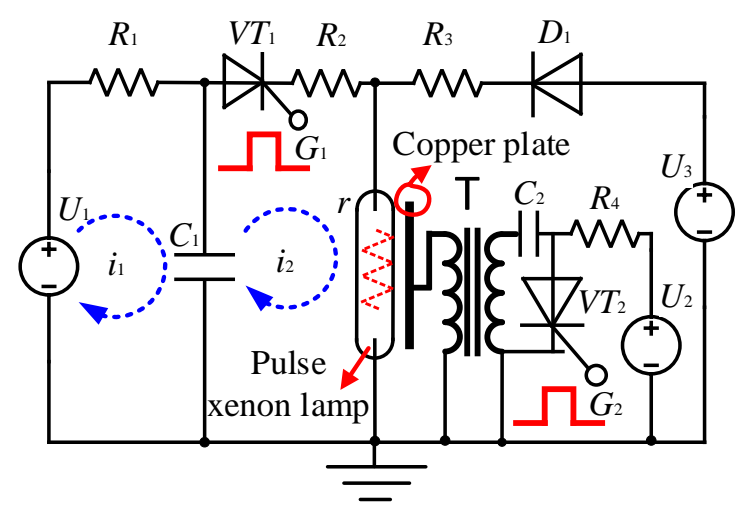

Figure 2.

asic circuit for high-voltage pulsed xenon discharge

At this time, the pulse xenon lamp can be approximated as a resistor, and the voltage $U_{3}$ is added to both ends of the pulse xenon lamp through the resistor $R_{3}$ and the diode $D_{1}$ to form a pre-combustion circuit; in the high-voltage discharge phase, the pulse xenon lamp is an arc discharge, and when the $\mathrm{VT}_{1}$ is turned off, the voltage $\mathrm{U}_{1}$ to the capacitor $\mathrm{C}_{1}$ is charged. When $\mathrm{VT}_{1}$ is turned on, the capacitor $C_{1}$ is discharged to the pulsed xenon lamp, so that the pulsed xenon lamp has an arc strobe phenomenon. In the high voltage discharge phase, the pre-combustion circuit always supplies a sustain current to the pulsed xenon

lamp. The TTL external control mode sends a rising edge of the pulse and turns on the pulse xenon lamp, and the falling edge turns off the pulse xenon lamp.

\section{Discharge control circuit}

According to Thevenin's theorem, which is to equip the two-terminal network into a voltage source in series with a resistor, the voltage and current of a complete charging process can be expressed as:

$$
\begin{array}{r}
i_{1}=\frac{U_{1}}{R_{1}} e^{-\frac{t}{R_{1} C_{1}}} \\
U_{c}=U_{1}\left(1-e^{-\frac{t}{R_{1} C_{1}}}\right)
\end{array}
$$

The voltage and current of the second complete discharge process can be expressed as:

$$
\begin{gathered}
U_{c}=\frac{U_{1}\left\{R_{2}+r+R_{1} \exp \left[-\frac{\left(R_{1}+R_{2}+r\right) t}{R_{1}\left(R_{2}+r\right) C_{1}}\right]\right\}}{R_{1}+R_{2}+r} \\
i_{c}=c \frac{d u}{d t}=\frac{U_{1}}{R_{2}+r} \exp \left[-\frac{\left(R_{1}+R_{2}+r\right) t}{R_{1}\left(R_{2}+r\right) C_{1}}\right] \\
\mathrm{W}=\mathrm{C}_{1} U_{c}{ }^{2} / 2
\end{gathered}
$$

The second incomplete charging voltage is:

$$
U_{c}=U_{1}\left(1-\frac{R_{1}}{R_{1}+R_{2}+r} e^{-\frac{t}{R_{1} C_{1}}}\right)
$$

See Figure 4 for the D-to-E charging voltage:

$$
U_{c 1}=U_{1}+\left(U_{D}-U_{1}\right) e^{-\frac{t}{R_{1} C_{1}}}
$$

The discharge voltage and current from point $\mathrm{E}$ to point F are:

$$
\begin{aligned}
U_{c}= & \frac{\left(R_{2}+r\right) U_{1}}{R_{1}+R_{2}+r}+\left[U_{E}-\frac{\left(R_{2}+r\right) U_{1}}{R_{1}+R_{2}+r}\right] \times \\
& \exp \left[-\frac{\left(R_{1}+R_{2}+r\right) t}{R_{1}\left(R_{2}+r\right) C_{1}}\right] \\
i_{c}=- & \frac{R_{1}+R_{2}+r}{R_{1}\left(R_{2}+r\right)} \times\left[U_{E}-\frac{\left(R_{2}+r\right) U_{1}}{R_{1}+R_{2}+r}\right] \times \\
& \exp \left[-\frac{\left(R_{1}+R_{2}+r\right) t}{R_{1}\left(R_{2}+r\right) C_{1}}\right]
\end{aligned}
$$

Figure 3 shows the relationship between the control pulse in the time domain, the $G_{1}$ and $G_{2}$ control signals, the high-voltage capacitor charging and discharging, and the correspondence relationship between the three figures in Figure 4(a)(b)(c). In turn, it can be showed the degree of influence of the speed uniformity of the lithography motion system on the energy level. 

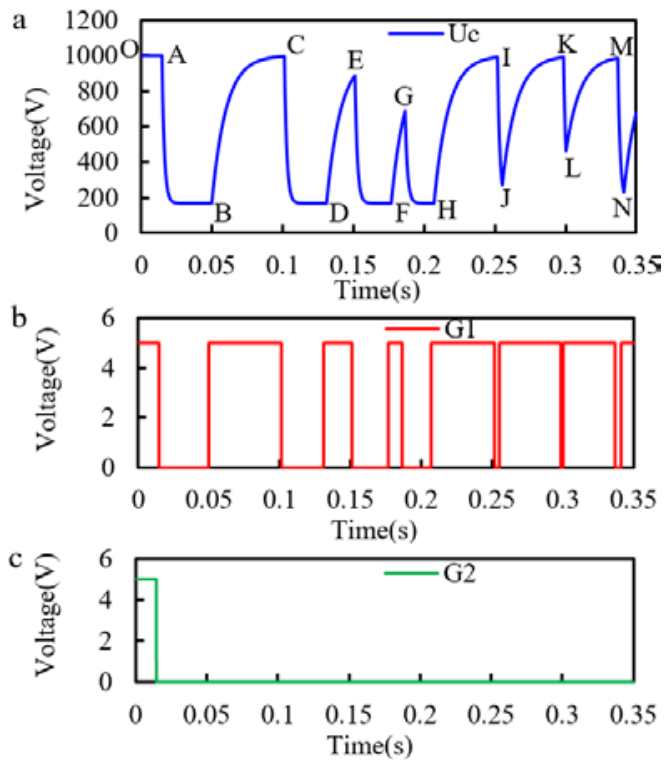

Figure 3.

ontrol pulse non-uniformity caused by changes in discharge energy (a) punch lamp voltage; (b) G1 control switch pulse; (c) G2 pre-ignition control switch pulse

\section{ANALYSIS OF TYPICAL EXAMPLES OF LITHOGRAPHY ENERGY}

The example shows the amount of energy change, set $\mathrm{R}_{1}=100 \Omega, \mathrm{R}_{2}=20 \Omega, \mathrm{r}=0.001 \Omega$, the variation of the discharge energy caused by the uneven control pulse is shown in Figure 3 . The $\mathrm{U}_{1} \mathrm{DC}$ voltage is $1000 \mathrm{~V}$, and the $\mathrm{T}$ transformer amplification ratio is 1:100. When the maximum pulse voltage of $\mathrm{U}_{2}$ is $50 \mathrm{~V}$, the pulse voltage of pre-ignition is $5000 \mathrm{~V}$ at most; the pulse voltage of $\mathrm{G}_{1}$ and $\mathrm{G}_{2}$ control signals is $5 \mathrm{~V} ; \mathrm{G}_{2}$ starts the glow discharge of the pulsed xenon lamp by one pulse; $R_{3}$ and $D_{1}$ form a freewheeling current. At this time, the channel of the pulse lighting is officially opened, and the pulse xenon lamp is equivalent to the path of the resistance $r$; due to the nonuniformity of the $\mathrm{G}_{1}$ switch change, the discharge voltage is obviously inconsistent, and also reflects the difference of the energy of each discharge. Figure 3 shows that the four situations are generated:

- Not fully charged, then discharged.

- $\quad$ Not fully charged, not fully discharged.

- Fully charged and then not fully discharged.

- Fully charged and fully discharged.

When the charging time is greater than $3 \tau_{1}\left(\tau_{1}\right.$ is the charging coefficient), when the discharge time is greater than $3 \tau_{2}\left(\tau_{2}\right.$ is the discharge coefficient), the energy fluctuation is less sensitive to time, that is, the fluctuation of the velocity is less required.

\section{A. Influence of pulsed xenon lamp resistance on lithography energy}

The resistance of the pulsed xenon lamp is affected by the diameter, material and process of the glass cover cylinder of the pulsed xenon lamp. In the calculation of the pulsed xenon lamp control circuit, the resistance of the pulsed xenon lamp has a great influence on the lithography energy, we use a typical resistance value to analyze it. The volt-ampere characteristics, as shown in Fig. 4 and Fig. 5, have a small change in volt-ampere characteristics below 1 $\Omega$, and as shown in Fig. 6 , the resistance value changes less than $1 \Omega$, and the change in exposure energy is small. When the resistance value exceeds or reaches $5 \Omega$. When the multiple is exceeded, the change in exposure energy can be clearly seen.

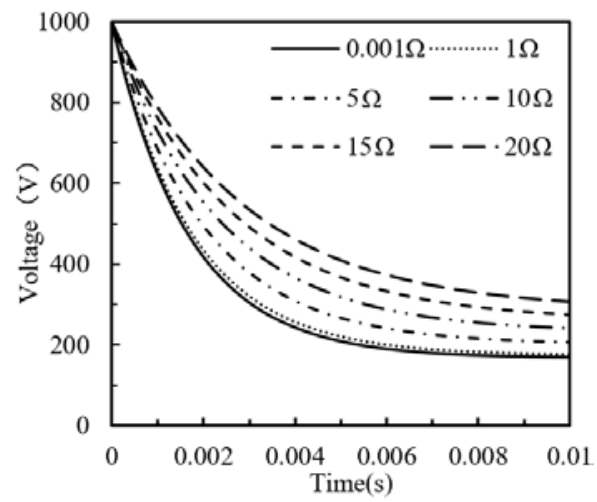

Figure 4.

oltages comparison under different pulse xenon lamp resistance

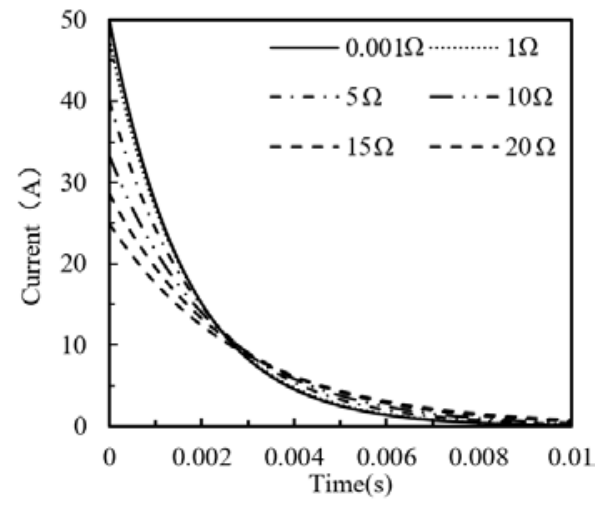

Figure 5.

urrents comparison under different pulse xenon lamp resistance

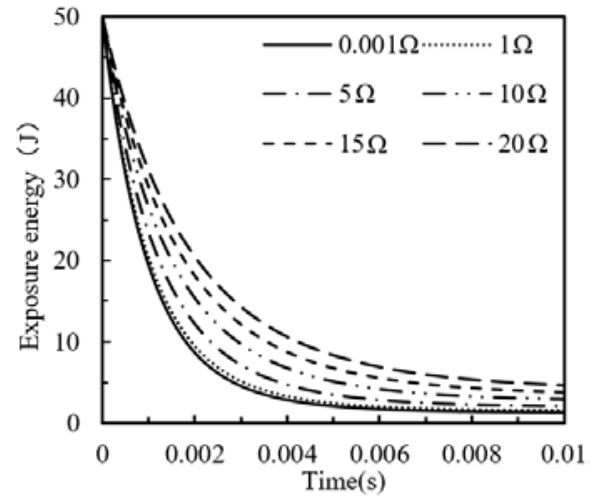

Figure 6.

xposure energy comparison under different pulse xenon lamp resistance

\section{B. Influence of exposure time delay error on lithography energy}

When the pulsed xenon lamp triggers the exposure, there is a time delay, which mainly includes the magnitude and fluctuation of the moving speed of the lithography machine, the system delay of the control system, the 
consumption of the exposure time, etc., which will cause the lithographic exposure energy to be uneven. Thereby affecting the accuracy of the grating lithography. From the volt-ampere characteristics of the control circuit, it can be seen that the pulsed xenon lamp discharge is below the $10 \mathrm{~ms}$ level; as shown in Figure 7, the pulsed xenon lamp discharge has completed 98\% of the energy control at $10 \mathrm{~ms}$, and at least $80 \%$ of the energy has been achieved at the $2 \mathrm{~ms}$ position. In order to reduce the influence of the exposure time delay errors on the lithography energy, the time delay error should be controlled within $2 \mathrm{~ms}$.

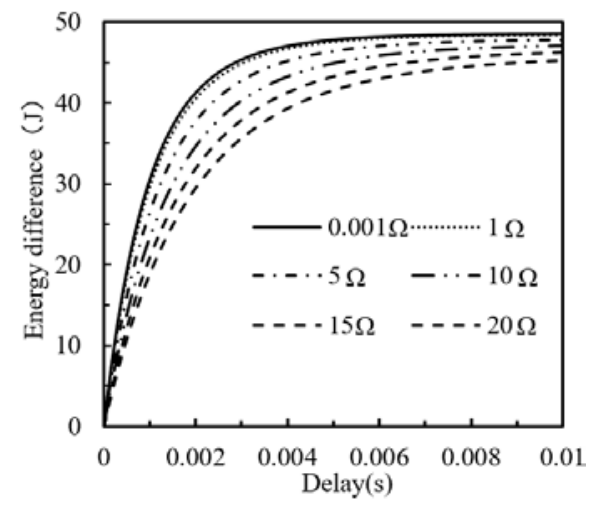

Figure 7.

xposure energy comparison for different exposure delay times

\section{Influence of lithographic energy control error on grating stripe accuracy caused by resistance factor}

In order to characterize the influence of the pulsed xenon lamp resistance on the grating stripe accuracy, the pitch value is $20 \mu \mathrm{m}$. As shown in Fig. 8, each pitch in the $\mathrm{X}$-axis direction represents the energy under a resistance value, wherein the resistance values are respectively 0.001 $\Omega, 0.01 . \Omega, 0.1 \Omega, 1-10 \Omega, 15 \Omega$ and $20 \Omega$. The time point is 2ms. The calculation lithography threshold model adopts the ideal plane segmentation model (see the pink plane in Fig. 8), and the grating stripe shown in Fig. 9 can be obtained by calculation, and the grating stripe pitch precision has a great influence on the resistance value greater than $5 \Omega$.

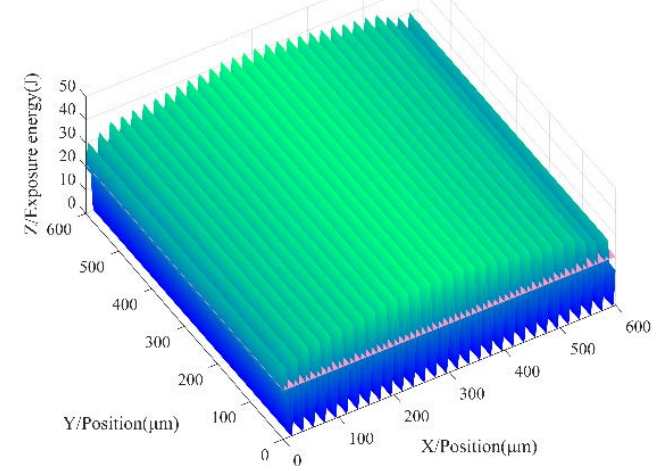

Figure 8.

xposure energy threshold under different pulsed xenon lamp resistance

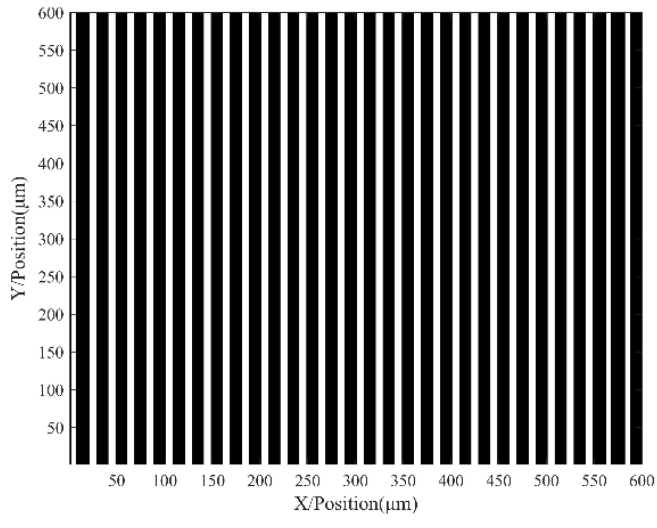

Figure 9.

rating strip formed by exposure energy threshold section corresponding to FIG.8

D. Influence of lithography energy control error on grating stripe accuracy caused by trigger time delay factor

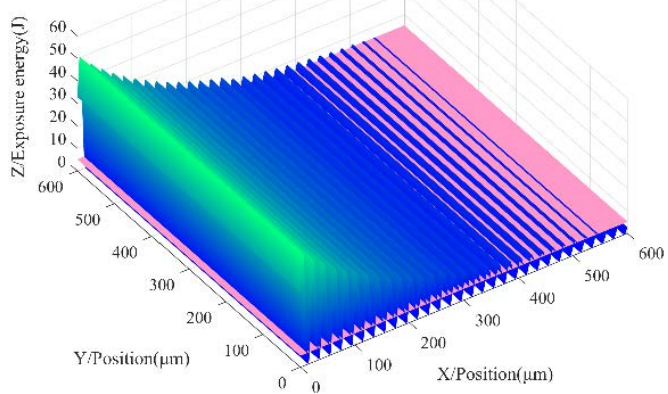

Figure 10.

xposure energy threshold at different trigger delay times

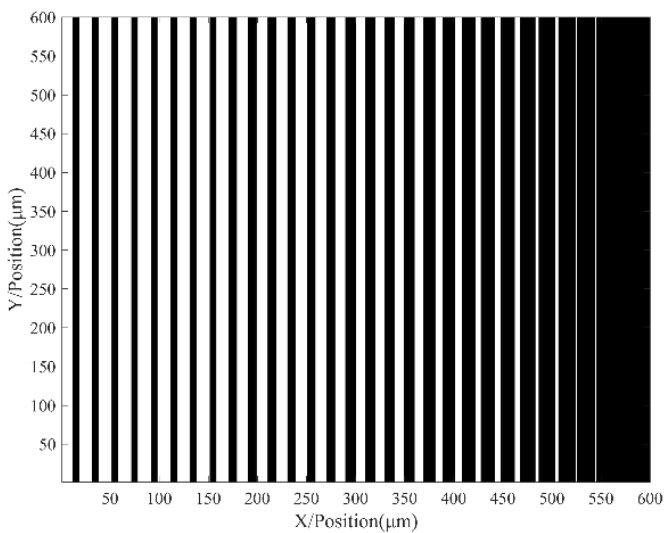

Figure 11.

rating strip formed by exposure energy threshold section corresponding to FIG.10

In order to characterize the influence of the trigger time E of the pulsed xenon lamp on the grating stripe accuracy, the pitch value is $20 \mu \mathrm{m}$, and the resistance value is $0.1 \Omega$. As shown in FIG. 10, each pitch in the $\mathrm{X}$-axis direction represents an energy of $0.1 \mathrm{~ms}$. The time range values are respectively $[0 \mathrm{~ms}, 0.3 \mathrm{~ms})$. The lithography threshold model 
is calculated by using the ideal plane segmentation model (see the pink plane in Fig. 10). The grating fringes shown in Fig. 11 can be obtained by calculation. The pitch accuracy of grating fringe has a great influence on the time delay error greater than $1 \mathrm{~ms}$.

\section{CONCLUSION}

Based on the charge and discharge circuit of the highvoltage pulsed xenon lamp power supply, the variation characteristics of charge and discharge voltage and current circuit and the charge-discharge coefficient are calculated and analyzed. The influence of control pulse error on energy is analyzed by typical examples. Conclusion: The resistance value of the pulsed xenon lamp should be controlled within $1 \Omega$, and the change in exposure energy is small. Based on the grating stripe of the lithography threshold model, the exposure time delay error is controlled within 2ms, achieving less than $80 \%$ energy release, reducing the impact on lithography energy. This paper will provide a theoretical reference for the directional energy control of grating scale precision scribing.

\section{ACKNOWLEDGMENT}

This project is supported by National Natural Science Foundation of China (Grant No.51475485), Open Laboratory of Key Disciplines of Modern Manufacturing Equipment and Instruments of Henan Higher Education
Institutions (2016MEI005) and Young Talents Innovation Ability Fund Project of Zhongyuan University of Technology (K2018QN002).

\section{REFERENCES}

[1] C. G. Cho, S. H. Song, S. M. Park, H. I. Park, S. R. Jang, and H. J. Ryoo, "A novel series-connected xenon lamp power supply system using pulse trigger with simmer circuits for pulsed light sintering application,” IEEE Transactions on Industrial Electronics, 2018: 1-1.

[2] S. Seung-Ho, C. Chan-Gi, P. Su-Mi, P. Hyun-Il, and R. Hong-Je, "Design and implementation of novel series trigger circuit for xenon flash lamp driver. IEEE Transactions on Plasma Science,” 2018, 1-7.

[3] S. Ruoyan, L. Jianjun, L. Haibing, W. Ruihua, and L. Hairong, "Research on continous preionized pulse xenon lamp power,” Applied Laser(1), 2015, 129-132.

[4] D. Ren, H. Zhao, C. Zhang, D. Yuan, J. Xi, and X. Zhu, et al, "Multi-repeated projection lithography for highprecision linear scale based on average homogenization effect, " Sensors, 2016, 16(4).

[5] D. Ren, J. Xi, Z. Li, B. Li, Z. Zhao, and H.Zhao, et al, “The alignment method for linear scale projection lithography based on ccd image analysis,” Proceedings, 2018, 2(3), 101 -

[6] D. Lin, H. Chen, Z. Liu, K. Dietrich, S. Kroker, and T. Kaesebier, et al, "Reducing rowland ghosts in diffraction gratings by dynamic exposure near-field holography," Optics letters, 2018, 43(4), 811. 